\title{
Roger Apéry: Un matemático muy radical
}

\author{
Vernor Arguedas T. \\ vernor.argueda@ucr.ac.cr \\ Universidad de Costa Rica
}

Recibido: Enero 5, 2017

Aceptado: Febrero 28, 2017

Resumen. En este artículo presentamos algunos aspectos de la vida de Roger Apéry, algunos datos biográficos, incluyendo su compromiso político sin dejar de lado sus intereses matemáticos y su papel fundamental en la matemática constructiva

Palabras clave: Función zeta de Riemann, irracionalidad de $\zeta(3)=\sum_{n=1}^{\infty} \frac{1}{n^{3}}$, matemática constructiva, partido Radical Socialista, ocupación nazi de Francia.

Abstract. In this article we present some aspects of the life of Roger Apéry, some biographical information, including his political commitment without leaving aside his mathematical interests and his fundamental role in constructive mathematics.

KeyWords: Riemann Zeta fuction, irrationality of $\zeta(3)=\sum_{n=1}^{\infty} \frac{1}{n^{3}}$, constructive mathematics Socialist Radical Party Socialista, nazi occupetation of France.

\subsection{Introducción}

Roger Apéry (14 noviembre 1916 - 18 diciembre 1994), fue un distinguido matemático francés- griego nació en Roen Francia y falleció en Caen Francia. En la lápida en donde se encuentras sus restos se ve: $1+\frac{1}{8}+\frac{1}{27}+\frac{1}{64}+\ldots \neq \frac{p}{q}$, que es el resultado más conocido de su autoría y al cual nos referiremos en este artículo.

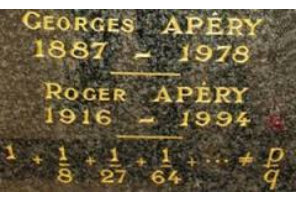




\subsection{Breve biografía de Apéry}

Su hijo Francois Apéry también matemático, escribió un artículo sobre su padre, lo que sigue a continuación es una adaptación de partes de su material. Su padre Georges Apéry, nació en Constantinopla, Grecia en 1887, llegó a Francia en 1903 para tratar de estudiar en la Ecole Nationale Supérieure d'Ingénieurs en Grenoble. Se incorporó al ejército como voluntario como un medio para obtener la ciudadanía francesa en 1914 . Participó en la campaña de los Dardanelos ( Turquía) en 1915 y regresó a Francia en un barco hospital luego de contraer tifoidea.

Le fue permitido viajar a Rouen en donde se casó con Justine Vander Cruyssen. Ella afrancesó su apellido a Delacroi y como no le gustaba el nombre Justine se llamaba a si misma Louise. En Rouen nació el único hijo Roger Apéry el 14 de noviembre de 1916. Su infancia transcurrió en Lille hasta 1926 cuando la familia se trasladó a París a un piso sin comodidades en la calle Goutte d'Or.

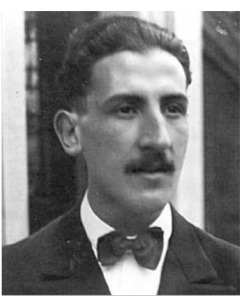

Su padre confiaba que la situación mejoraría y se pudieran cambiar a un lugar mejor. Pero la crisis de 1929 lo afectó - como a muchos- perdió su trabajo de ingeniero y como era considerado muy viejo no pudo conseguir trabajo en su campo.

Trabajó como guardia en el ministerio de los antiguos combatientes (Ministére des Anciens Combattants). Su madre Louise daba clases de piano aquí y allá, pero sin esperanza de días mejores. Así que permanecieron en el piso que tenía un baño compartido con otros inquilinos, no tenía gas, y la calefacción provenía de una vieja cocina de hierro, el alumbrado era por medio de lámparas de gas hasta después de la segunda guerra mundial, Georg Apéry hizo él mismo la instalación eléctrica.

Desde luego que un muchacho inteligente se esforzaría para salir de este ambiente. Animado por sus padres se esforzó en tener éxito académico. Su única experiencia en trabajos manuales fue en la clase de trabajo en madera, la cual concluyó con un tablero roto en la cabeza de un compañero. Amante del ajedrez, jugaba con los estudiantes de la Universidad de Caen y en 1966 era el presidente del recién formado Círculo de Alekhine. Estaba algo orgulloso de haberle ganado una partida unos años después al campeón de Holanda durante unas simultáneas ( durante una actividad académica en Antwerp).

Su vida romántica fue conflictiva. Se casó en 1947 y tuvo tres hijos, pero la vida tensa y amarga en el hogar terminó en divorcio en 1971, se casó por segunda vez en 1972 y se divorció de nuevo en 1977. Pareciera que no pudo reconciliar la vida familiar, la investigación matemática y su activismo político. De su madre heredó el amor a la música, ella le enseñó suficiente piano, por lo que él consideró a sus 18 años hacer una carrera como músico, pero sus padres lo desalentaron, así que se enrumbó hacia las matemáticas. 
De su padre el obtuvo el amor a su patria, la defensa intransigente de las ideas republicanas encarnadas en Clemenceau y tal vez la necesidad de tener siempre razón. Nunca aprendió a hacer concesiones incluso cuando esto le hubiera permitido avanzar en su causa.

Esto y su resistencia ocasional al decoro social básico posiblemente contribuyeron a su nada conformista carrera (Los estudiantes en Caen contaban de las veces que un conserje interrumpía su clase porque había un niño llorando en su bicicleta a motor, se le había olvidado sacar a su hijo del asiento de bebé.)

Fue sometido a una operación de colon, pera era Parkinson lo que tenía, se le diagnosticó en 1977 y fue la causa de su fallecimiento. La enfermedad mermó sus habilidades físicas, lo privó de escribir o de tocar piano y finalmente disminuyó sus capacidades intelectuales. Murió el 18 de diciembre de 1994 en Caen.

Roger era un estudiante solitario y dedicado en la escuela, llevando a su casa premio tras premio. El dejó el Lycée Faidherbe de Lille in 1926 después de haberse saltado dos grados y ganó una reputación de estudiante estrella en los liceos Ledru-Rollin y Louis-le-Grand en Paris. Él se apasionaba por la historia y sobre todo las matemáticas, no mucho en las lenguas pues él hablaba un poco de alemán y de italiano.

A los 8 años de edad se interesó en la "preuve .par neuf" ( método empírico para realizar operaciones aritméticas, en desuso actualmente) a los 12 se interesó en los postulados de Euclides , a los 16 aprendió de un profesor que tuvo que la razón cruzada de cuatro tangentes desde un punto a un plano cúbico no singular es un invariante proyectivo de la curva y así empezó su atracción por la geometría algebraica.

Quedó en el tercer lugar en el concurso nacional de matemáticas de 1932. Al año siguiente fue superado por una nariz por un estudiante fuera de Paris: Gustave Choquet, así que se tuvo que conformar con un segundo lugar en matemáticas y una mención de honor en física.

En 1936 recibió su diploma de estudios superiores en geometría inversiva dirigido por E.Cartan.

Hizo el exámen de agregación y en la prueba escrita de análisis lo examinó Jean Dieudonné quien quedó impresionado por las capacidades mostradas por Apéry, se hicieron amigos tiempo después. Dieudonné cuenta que fue la única vez que estuvo en un jurado de agregación y escribió un ejercicio especialmente difícil, quedó impresionado con dos estudiantes Apéry y Jacqueline Ferrand.

En setiembre de 1939 fue llamado a tomar parte en el ejército francés en la guerra contra Alemania y tomado prisionero de guerra en junio de 1940, repatriado con pleuresía en junio del 41, hospitalizado hasta agosto de ese año. Durante su cautiverio se mantuvo en contacto con Lucien Godeaux y Francesco Severi de quienes recibió varios artículos por medio de la Cruz Roja.

Después recibió de George Bruhat director de École Normale Supérieure una beca de investigación del CNRS (Centre national de la recherche scientifique), en 1943 Elie Cartan quien había intervenido con las autoridades para que fuera repatriado, le ofreció un puesto de asistente en la Sorbonne y así retomó a su producción matemática. 
Escribió su tesis doctoral en geometría algebraica bajo la dirección de Paul Dubreil y René Garnier en 1947 y fue el maestro de conferencias más joven en Francia en ese año. Fue nombrado profesor en Caen en 1949, su especialidad de geometría algebraica sobre los números complejo se fue moviendo hacia geometría algebraica sobre los racionales y hacia la teoría de números.

Ël nunca se sumó a la teoría de esquemas que Grothendieck lanzó al principio de los sesenta del siglo pasado. Sin embargo en el verano de 1964 en el Séminaire de mathématiques supérieures en Montreal, Dieudonné quien estaba exponiendo la geometría algebraica de esquemas llamó a Apéry para que de manera simultánea hiciera la traducción en el lenguaje clásico.

El momento más importante de su vida como matemático fue cuando con más de 60 años de edad en 1979 demostró que $Z(3)$ es un irracional.

La función zeta de Riemann se define como: $\zeta(s)=\sum_{n=1}^{\infty} \frac{1}{n^{s}}$ y $\zeta(3)=\sum_{n=1}^{\infty} \frac{1}{n^{3}}$

\subsection{Roger Apéry un político comprometido}

A la par de su trabajo matemático Apéry desarrolló una intensa actividad política desde su juventud bajo la bandera del radicalismo en donde mantuvo su posición anticlerical, Su actividad política se remonta a los disturbios de febrero de 1934 cuando se unió al Partido Radical Socialista de Camille Pelletan. En las elecciones de 1936 este partido obtuvo dos escaños clasificado bajo "izquierda mixta" en la Asamblea Legislativa del Frente Popular.

En 1938 Apéry firmó la petición de Éduard Herriot en contra de los acuerdos de Munich y devolvió su membresía en el Partido Radical, se cambió al movimiento radical de Édouard Daladier. Después de su regreso del campo de prisioneros de guerra en 1941, regresó a su actividad política bajo la influencia de su amigo comunista Marrot, Esto a pesar de su desilusión con el Partido Comunista Francés con respecto al pacto de no agresión entre la Alemania nazi y la Unión Soviética en 1939. Participó en la red de Louis Fernand Marty con el seudónimo de Arthur Morin. Llegó a ser Director del Frente Nacional un movimiento de resistencia en la École Normale Supérieure después de que su predecesor Mark Zamansky fuese arrestado y deportado al campo de concentración de Mauthausen.

Apéry organizó una demostración en contra del arresto de George Bruhat y Jean Baillou, participó en actividades en contra de la obligación de que la población judía usara la estrella amarilla . Participó en la distribución de material clandestino, documentación falsa de identidad y armas para la resistencia, el peligro estuvo siempre presente en este periodo incluso a niveles de riesgo extremo.

Algunos de su colegas murieron en campos de concentración nazis como Bruhat. Apéry recibió igual que se padre al finalizar la primera guerra mundial la Cruz del Combatiente Voluntario (Croix de Combattant Volontaire). Fue notoria su participación en el caso Audin. Después de 1948 se decepcionó de los Comunistas por su apoyo a la biología de Lysenko. En 1952 , en actividades públicas se manifestó 
en contra de la imposición de una línea marxista en las matemáticas en Francia.

Apoyó siempre a Mendez-France contra de Gaulleyt mantuvo la tesis de que el norte de África debía ser parte integral de Francia, para él fue imperdonable la conducta de Gaulle en 1962 de abandonar Algeria en las peores condiciones posibles.

En 1969 abandonó el movimiento radical de manera definitiva al considerar que el espíritu republicano había sido dejado de lado por esa corriente política.

Cuando el General de Gaulle falleció, dejó Apéry toda actividad política al considerar que las ideas republicanas ya no estaban amenazadas.

\subsection{Las matemáticas para Apéry}

Polemizó con el grupo Bourbaki y no aceptó formar parte del mismo cuando Dieudonné le invitó. El manifiesto Mathématique constructive (Apéry 1982 [7]) ha sido traducido a varias lenguas incluso al castellano y expone con gran claridad el carácter constructivo e intuicionista de esta disciplina en contraposición a la matemática sólo axiomática de Hilbert o Bourbaki.

Me permito transcribir por su importancia en su original en francés una conferencia de Apéry sobre el tema. Él fue un defensor de la teoría inicial de Categorías, al estilo de Saunder Mac Lane (suposición mía).

\section{Mathématique constructive}

Par Roger Apéry, in Penser les mathématiques, Séminaire de philosophie et mathématiques de l'Ecole Normale Supérieure (J. Dieudonné, M. Loi, R. Thom). Éditions du Seuil, 1982, pp. 58-72.

"Qui veut tuer son chien l'accuse de la rage." Pour combattre une dissidence religieuse, philosophique ou politique, un pouvoir commence toujours par la discréditer, en lui ôtant son caractère de doctrine soutenue par des chercheurs de bonne volonté attachés à leur conviction intime par des arguments solides pour la présenter comme une entreprise criminelle (hérétique, asociale) vouée à la disparition. Selon la caricature présentée par ses adversaires sous le nom d'intuitionnisme, la conception constructive détruirait une grande part de la mathématique classique, notamment l'axiome de choix et ses conséquences; contrairement au caractère objectif de la science, elle adopterait comme critère de vérité l'intuition particulière de chaque mathématicien, elle ne serait qu'une singularité historique, liée à une métaphysique particulière destinée à disparaître ; elle n'exprimerait que l'angoisse de quelques mathématiciens. À défaut de convaincre, ce texte pourra dissiper des malentendus : nous montrons que la conception constructive ne mutile pas la mathématique classique, mais au contraire l'enrichit. Nous de traitons pas de l'axiome de choix dont la discussion n'est pas essentielle. Nous indiquons les critères objectifs de preuve utilisés par les mathématiciens constructifs. Enfin, aucun argument solide ne permet d'affirmer que L. Kronecker, H. Poincaré ou H. Weyl étaient plus angoissés que Cantor, Hilbert ou Russell. 


\section{Les principales philosophie des mathématiques}

Le platonisme mathématique (Bolzano, Frege, Cantor, Russell)

Comme toute science, la mathématique traite d'une réalité indépendante de chaque mathématicien particulier: la géométrie étudie des droites et des cercles idéaux, non des traits et des ronds dessinés. La conception platonicienne reporte sur le monde mathématique le désir d'absolu et d'éternité de l'esprit humain.

Les principales affirmations du platonisme mathématique sont les suivantes :

$1^{\circ}$ Toute question mathématique concerne des objets aussi réels (et même plus réels) que les astres, les animaux ou les végétaux; elle a donc une réponse (éventuellement inconnue) affirmative ou négative: c'est la logique bivalente et son corollaire, le principe du tiers exclus.

$2^{\circ}$ La notion d'ensemble, définie par Cantor comme " un groupement en un tout d'objets bien distincts de notre intuition et de notre pensée (1)", est simple, primitive et constitue à elle seule le fondement de toutes les mathématiques. Par exemple, le nombre 1 est défini par Russell comme l'ensemble de tous les ensembles $E$ non vides tels que $x \in E$ et $y \in E \Rightarrow x=y$.

$3^{\circ}$ L'existence simultanée de tous les êtres mathématiques exige de traiter comme une unité achevée tout ensemble infini; c'est la doctrine de l'infini actuel soutenue par Leibniz et étendue par Cantor pour des raisons métaphysique.

"Je suis tellement pour l'infini actuel qu'au lieu d'admettre que la nature l'abhorre, je tiens qu'elle l'affecte partout, pour mieux marquer la perfection de son auteur. Ainsi, je crois qu'il n'y a aucune partie de la matière qui ne soit, je ne dis pas divisible, mais actuellement divisée, et, par conséquent, la moindre particule doit être considérée comme un monde plein d'une infinité de créatures différentes" (Leibniz).

"Sans un petit grain de métaphysique, il n'est pas possible, à mon avis, de fonder une science exacte. La métaphysique telle que je la conçois est la science de ce qui est, c'est-à-dire de ce qui existe, donc du monde tel qu'il est en soi et pas tel qu'il nous apparaît" (Cantor).

"La plus haute perfection de Dieu est la possibilité de créer un ensemble infini et son immense bonté le conduit à le créer" (Cantor).

Les difficultés de la théorie cantorienne se manifestèrent sous forme d'antinomies. L'édifice s'effondra quand Russell montra que le monde cantorien lui-même, c'est-à-dire l'ensemble de tous les ensembles, est contradictoire.

\section{Le formalisme}

Le formalisme, conçu par Hilbert et poussé à l'extrême par Bourbaki, veut créer un ordre mathématique dont les commandements sont les suivants:

$1^{\circ}$ Que la réglementation des méthodes autorisées soit suffisamment rigide pour empêcher toute discussion.

$2^{\circ}$ Que l'on ne rencontre pas de contradictions et, en particulier, que l'on évite les paradoxes. 
$3^{\circ}$ que l'on conserve la mythologie du transfini qu?Hilbert appelle le "paradis créé pour nous par Cantor".

Ce objectif est atteint par la méthode suivante :

$1^{\circ}$ Rejeter $1^{\prime}$ ordre ancien en lui reprochant simultanément d'être trop libéral (mot d'ordre: "A bas Euclide", lancé par Bourbaki) et d'être autoritaire (Hilbert traitant Kronecker de Verbotsdiktator).

$2^{\circ}$ Considérer comme infranchissable le fossé entre les mathématiques et les autres disciplines.

$3^{\circ}$ Attribuer la réussite de l'application des mathématiques aux autres sciences à $1^{\prime}$ "harmonie préétablie" (Leibniz) ou à un "miracle" (Bourbaki).

$4^{\circ}$ Réduire la mathématique au texte écrit, ce qui rejette à la fois comme inexistant le monde platonicien et comme épiphénomène la pensée du mathématicien.

$5^{\circ}$ Refuser comme dénués de sens les concepts d'espace, de temps, de liberté.

$6^{\circ}$ "Imposer au domaine mathématique des bornes en grande partie arbitraires" (Bourbaki, théorie des ensembles, p. E. IV. 67).

$7^{\circ}$ Pratiquer le double langage ${ }^{2}$, d'une part en laissant croire qu'une seule école possède la "bonne mathématique" et en considérant les mathématiques comme un simple jeu, ou, par exemple, "les mots " il existe" dans un texte formalisé n'ont pas plus de "signification" que les autres, et [où] il n'y a pas à considérer d'autres types d' "existence" dans les démonstrations formalisé 3 (Bourbaki).

$8^{\circ}$ Extirper l'intuition, notamment en refusant l'usage des figures dans l'enseignement.

$9^{\circ}$ Considérer comme "métamathématiques" toutes les questions gênantes sur la structure des mathématiques.

$10^{\circ}$ Uniformiser les esprits par l'enseignement des "mathématiques modernes", où on laisse croire aux enfants qu'entourer des petits objets par une ficelle est une activité mathématique au lieu de leur apprendre à compter, à calculer et à examiner les propriétés des figures.

$11^{\circ}$ Créer un Dieu mathématique à plusieurs personnes qui tente d'assurer son immortalité en renouvelant périodiquement ses membres et qui assure l'unité de la communauté mathématique en révélant périodiquement les bonnes définitions et les bonnesthéories.

Hilbert espérait démontrer la cohérence de sa conception, mais Gödel, en montrant que toute théorie contenant au moins l'arithmétique élémentaire contient des résultats vrais mais non démontrables par axiomatique, mettait en évidence l'échec du formalisme hilbertien. Il faut distinguer entre la méthode formaliste et la philosophie formaliste. Tous les logiciens utilisent la méthode formaliste pour préciser les types de déductions valables; la philosophie formaliste considère le texte formalisé non comme un outil commode, mais comme la seule réalité mathématique (les physiciens connaissent une distinction analogue entre la méthode positive, qui est la méthode de tous, et la positivisme, qui est la philosophie de quelques-uns). On fixe une théorie mathématique en indiquant les propriétés de départ (axiomes) et les règles de déductions admises. Le scepticisme vis-à-vis de certains principe traduit généralement un dogmatisme sous-jacent qui refuse d'expliciter ses propres principes et de les laisser critiquer. Ainsi les formalistes, qui soumettent à une critique poussée les propriétés mathématiques élémentaires, avalent sans examen les règles traditionnelles de logique, en refusent la mise en cause, oublient que ces règles, issues de l'expérience courante comme la géométrie euclidienne, n'ont comme elle qu?un champ d'application limité. Il ne pas sont pas sûrs de la vérité de $2+2=4$, considèrent comme un axiome gratuit, donc susceptible d'être rejeté, qu'en 
enlevant le dernier signe de deux suites isomorphes en obtient des suites isomorphes, ce qui entraîne $1^{\prime}$ "axiome de Peano" selon lequel deux nombres naturels ayant mêmes successeurs sont égaux. Par contre, ils considèrent comme évident et incontestable l'axiome logique de Peirce selon lequel, quelles que soient les propositions $\mathrm{p}, \mathrm{q}$, on peut déduire de la proposition $(p \Rightarrow q) \Rightarrow p$ la proposition $\mathrm{p}$; toute mise en cause du principe du tiers exclu leur apparaît non comme une opinion discutable, mais comme un scandale intolérable.

\section{Le mathématicien idéal selon le constructivisme}

Selon la conception constructive, il n'y a pas de mathématique sans mathématicien. En tant qu'êtres de raison, les êtres mathématiques n'existent que dans la pensée du mathématicien et non dans un monde platonicien indépendant de l'esprit humain ; quant aux textes mathématiques, ils ne prennent un sens que par une interprétation qui exige un lecteur connaissant le langage utilisé par l'auteur du texte. Le mathématicien idéal se définit par un certain comportement mental dont la pensée effective du mathématicien concret n'est qu'une image approchée.

Les hypothèses nécessaires pour l'activité mathématique sont les suivantes :

$1^{\circ}$ On peut toujours ajouter un nouveau signe à une formule ; en particulier, après tout nombre entier, on peut en considérer un autre.

$2^{\circ}$ Le mathématicien raisonne toujours en appliquant des règles de déductions explicitement précisées.

$3^{\circ}$ Tout résultat démontré est définitivement acquis.

$4^{\circ}$ L'aptitude à tirer des déductions ne se détériore pas et ne s'améliore pas.

Toutes ces propriétés supposent que le mathématicien satisfasse aux conditions suivantes :

$1^{\circ}$ Il est immortel, c'est-à-dire qu'il peut toujours continuer un calcul inachevé.

$2^{\circ}$ Il est imperméable à la douleur, aux passions, aux souffrances, ce qui maintient la rigueur nécessaire de sa pensée.

$3^{\circ}$ Grâce à une mémoire parfaite, il n'oublie ni ne déforme aucun résultat acquis.

$4^{\circ}$ Il ne se fatigue pas et effectue des performances sans entraînement préalable.

Les mathématiciens suppléent à leur différence évidente avec le mathématicien idéal :

$1^{\circ}$ Par l'entraide : l'erreur qui échappe à un mathématicien peut être décelée par un autre.

$2^{\circ}$ Par les mémoires mécaniques (textes manuscrits ou imprimés) qui suppléent aux défaillances de la mémoire individuelle.

$3^{\circ}$ Par les machines à calculer qui leur permettent d'effectuer en un temps raisonnable des calculs que, sans machine, leur vie n'aurait pas suffi à achever.

S'il extrapole la réalité, le mathématicien constructif refuse les hypothèses fantastiques des platoniciens. En effet :

$1^{\circ}$ Il ne se croit pas éternel : l'activité mathématique a eu un commencement.

$2^{\circ} \mathrm{Il}$ croit que les êtres mathématiques sont des êtres de raison; ils apparaissent au moment où le mathématicien les définit et non antérieurement à tout mathématicien. 
$3^{\circ}$ Il constate que la mathématique se déroule dans le temps. Un raisonnement est une méthode pour montrer que si certaines affirmations sont supposées vraies avant, d'autres deviennent vraies après.

$4^{\circ}$ Son immortalité lui permet d'atteindre des nombres aussi grands qu'il veut, mais pas de définir tous les nombres ; il croit à l'infini potentiel, pas à l'infini actuel.

Alors que les mathématiciens idéaux sont interchangeables, les mathématiciens concrets sont divers, et chacun d'entre eux se modifie dans le temps ; cette diversité entraîne dans l'activité mathématique une part subjective qui ne peut être supprimée. Cette part subjective se manifeste dans la création, dans l'apprentissage, dans la reproduction. Malgré son importance, ce n'est pas elle qui constitue la différence entre mathématique statique et mathématique constructive.

\section{Mathématique et durée}

Comme le platonicien et contrairement au formaliste, le mathématicien constructif reconnaît une certaine réalité aux objets mathématiques, mais les différencie essentiellement des objets matériels, en ne leur attribuant que les propriétés susceptibles de démonstration. Une distinction analogue différencie les héros de roman des personnages historiques. Une question concernant Vercingétorix admet une réponse, même si elle échappe à nos moyens d'investigation ; la même question concernant Don Quichotte n'a pas de réponse si celle-ci ne peut être déduite des affirmations du roman de Cervantès. En revanche, l'existence d'ensembles plus nombreux que l'ensemble des entiers et moins nombreux que l'ensemble des réels n'a pas de réponse, car, comme Paul Cohen l'a démontré, ni cette existence ni sa négation ne peuvent être déduites des définitions usuelles des réels : l'ensemble des réels, comme Don Quichotte, est un être essentiellement incomplet.

Le mathématicien constructif refuse le tabou philosophique interdisant de parler de temps et de liberté, car toute activité mathématique exige un esprit libre opérant dans le temps. Laissant au moraliste le temps irréversible, ce fameux " temps perdu " qui ne se rattrape jamais, les mathématiciens, comme les musiciens, utilisent un temps reproductible. Une statue, un tableau, un monument, essentiellement situés dans l'espace, se maintiennent par eux-mêmes ; les forces extérieures peuvent les user ou les détruire, mais ne sont pas nécessaires à leur maintient ; l'examen de leurs diverses parties s'opère selon un ordre arbitraire et pendant une durée arbitraire. Au contraire, la musique se situe essentiellement dans le temps. Une mélodie n'est pas un ensemble, mais une suite de note subtilement reliées : contrairement aux monuments qui perdurent, la mélodie disparaît ; pour réapparaître, elle doit être reproduite ; elle est conservée par des procédés de mémorisation artificiels (partitions musicales, disques). Nous connaissons les outils ou les dessins de nos ancêtres préhistoriques, nous ignorons leurs paroles ou éventuellement leur chants.

De même, un raisonnement mathématique, essentiellement fragile, doit être refait pour être compris : un texte mathématique se lie la plume à la main. Bien que la durée semble moins contraignante qu'en musique, l'examen d'un raisonnement mathématique exige d'embrasser simultanément à chaque étape les prémisses, la conclusion, la règle de raisonnement utilisée ; une compréhension authentique s'adresse à l'ensemble des articulations du raisonnement, de façon que le résultat apparaissent dû à une méthode applicable à d'autres problèmes et non à un heureux hasard. Schématiquement, l'activité mathématique comporte deux phases, caractérisées par la boutade : $5 \%$ d'inspiration, $95 \%$ de transpiration. 
Dans la première phase, l'activité mentale, subjective, indépendante du langage, étroitement liée à la durée intuitive. Malgré ses deux faiblesses (fugacité et incommunicabilité), cette phase constitue l'activité mathématique authentique. Dans la seconde phase, le mathématicien note, formaliste, traduit (partiellement) sont intuitions en terme communicables ; chacun peut examiner ses résultats devenus objectifs. Les diverses exécutions d'une oeuvre musicale ne sont jamais rigoureusement identiques, elles dépendent de la personnalité du chef d'orchestre. De même, la reproduction d'un raisonnement contient une part subjective irréductible ; en rappelant qu'un chien dévorant une oie emmagasine de la graisse de chien et non de la graisse d'oie, H. Poincaré illustre la nécessité pour chacun d'incorporer à sa propre personnalité toute connaissance extérieure. Celui qui possède des textes mathématiques dont il ne comprend pas l'articulation ne possède rien.

\section{Quelques outils et concepts des mathématiques constructives}

\section{Nombres naturels}

Comme Bourbaki (Théorie des ensembles, chap. I. §1), nous commençons les mathématiques par l'étude d'assemblages de signes extraits d'un alphabet; un tel assemblage est une suite, non un ensemble. Tous les mathématiciens s'accordent sur la philosophie des signes : tout signe est indestructible, peut être reproduit sans changement ni usure autant de fois qu'on le désire, peut servir à construire des formules de longueur arbitraire. Un texte mathématique se présente comme une suite d'arguments correctement déduits, non comme un ensemble d'affirmations en vrac. Les assemblage construits avec un alphabet à un seul signe, noté | , sont les nombres naturels. L'assemblage vide est doté 0 , les assemblages $||,||||$, , sont notés respectivement $1,2,3$.

Devant une question mathématique élémentaire, par exemple rechercher s'il existe un entier naturel qui vérifie une propriété simple (c'est-à-dire une propriété qui peut être effectivement décidée pour chaque entier donné), trois situations se rencontrent pratiquement :

a.) on connaît une solution ;

b.) on peut montrer que l'existence d'une solution conduit à une contradiction ;

c.) on ne sait pas.

Les mathématiciens s'accordent sur la réponse au problème dans les cas $a$ ) et $b$ ). Les différences d'attitude apparaissent dans le cas $c$ ), qui est le plus intéressant (il recouvre tous les problèmes mathématiques non résolus, c'est-à-dire toute la mathématique vivante).

Une attitude empiriste n'admettrait que des réponse à des questions déjà tranchées. L'attitude statique considère notre incapacité de répondre comme infinité humaine mais admet une réponse "en soi".

L'attitude constructive est intermédiaire. Devant une proposition $p$ non tranchée, le mathématicien constructif ne refuse pas toujours de poser $p$ ou non $p$.

Mais il n'admet la validité de cette expression logique (application du principe du tiers exclu à l'énoncé $p$ ) que s'il possède un algorithme qui au bout d'un nombre fini d'étapes permettra de trancher, quelle que soit par ailleurs la longueur de l'algorithme. Comme un tel algorithme n'existe pas toujours, il y a donc des énoncés auxquels le principe du tiers exclu de s'applique pas. 
Une suite d'entiers (ou de rationnels) est un processus qui associe à chaque nombre naturel un entier (ou un rationnel) $u(n)$ noté encore $u_{n}$. Si c'est à l'occasion des suites d'entiers qu'apparaît le point crucial du débat : infini actuel ou infini potentiel. Selon la conception constructive, une suite infinie, par exemple la suite des nombres naturels, n'est jamais finie, c'est-à-dire n'est jamais achevée : après tout nombre entier, on peut en construire un autre ; c'est la conception de l'infini potentiel qui fut soutenue par Gauss et Poincaré. Il n'existe pas d'ensemble effectivement infini. Une propriété qui exige de tester tous les éléments d'une suite ne relève pas de la loi du tiers exclu. En appelle suite fugace une suite dont tous les éléments effectivement calculés sont nuls, mais dont on ignore si le calcul de nouveaux éléments donnera toujours des zéros. Des problèmes importants posés aux mathématiciens équivalent à la question de savoir si une suite fugace est nulle ou non (conjecture de Fermat ou de Riemann). La comparaison de deux suites $u(n)$ et $v(n)$ revient à examiner si la suite $|u(n)-v(n)|$ est nulle. L'existence de suites fugaces montre que, contrairement aux nombres (naturels, entiers relatifs ou rationnels), deux suites ne sont pas nécessairement égales ou inégales.

\section{Logique constructive}

Platoniciens et formalistes utilisent une même logique " classique ", que nous comparons à la logique constructive. On distingue la logique propositionnelle qui examine les propositions complexes bâties à l'aide de propositions élémentaires et de connecteurs (généralement, $\wedge$, $\vee, \Rightarrow, \Leftrightarrow$ ) et la logique des prédicats (à une ou plusieurs places) qui utilise notamment les quantificateurs $\forall, \exists$. La logique classique n'a besoin que des connecteurs $\wedge$, $\neg$ et du quantificateur $\forall$; les connecteurs $\vee$, $\Rightarrow$ et le quantificateur $\exists$ sont, selon les classiques, des abréviations :

$p \vee q$ signifie $\neg(\neg p \wedge \neg q)$

$p \Rightarrow q$ signifie $\neg(p \wedge \neg q)$

$\exists x$ P $x$ signifie $\neg \forall x \neg \mathrm{P} x$.

Certains énoncés complexes bâtis avec des propositions élémentaires indéterminées constituent des thèses logiques, c'est-à-dire sont considérés comme " vrais " quelles que soient les propositions considérées, par exemple :

$p \Rightarrow(q \Rightarrow p)$

Toutes les thèses de la logique classique sont vraies en logique constructive : autrement dit, contrairement à la légende, la mathématique constructive ajoute quelque chose à la mathématique classique et ne lui retranche rien. L'originalité de la logique constructive est l'introduction de connecteurs que nous noterons $\overline{\mathrm{V}}, \bar{\Rightarrow}$, et d'un quantificateur que nous noterons $\bar{\exists}$, qui ne peuvent s'exprimer en logique classique.

$p \bar{\vee} q$ signifie : il existe un procédé régulier qui permet soit d'affirmer $p$, soit d'affirmer $q$.

$\bar{\exists} P(x)$ signifie : il existe un procédé régulier qui permet de construire un élément vérifiant la propriété $P$. 
$C^{\prime}$ est un faux problème de demander qui a raison, du classique affirmant la thèse $p \vee \neg p$, qui n'est pour lui que l'abréviation de $\neg(\neg p \wedge \neg \neg p)$ et se déduit du principe de non-contradiction, et du constructiviste qui nie la thèse $p \bar{V} \neg p$, qui supposerait une méthode pour résoudre tous les problèmes mathématiques. En toute rigueur, le mathématicien classique qui accepte le principe du tiers exclu et le mathématicien constructif qui le rejette ne parlent pas de la même chose. Même avec les connecteurs constructifs, il existe des propositions pour lesquels le tiers exclus s'applique. Les arguments mettant en cause le flou des affirmations courantes ne justifient pas le rejet du tiers exclu : la mathématique exige l'existence d'énoncés que l'on puisse nécessairement affirmer ou nier. Le tiers exclu cesse de s'appliquer pour des propositions dont la démonstration ou la réfutation exigerait de décider d'une infinité de questions. Il arrive qu'une méthode adéquate permette de trancher un problème par un raisonnement fini, mais ce n'est pas toujours le cas.

$\bar{\nabla}, \rightrightarrows$, et d'un quantificateur que nous noterons $\bar{\exists}$, n'acquièrent un sens précis qu'après une définition précise d'un procédé régulier. Les diverses définitions de la calculabilité tentées par les logiciens se sont révélées équivalentes ${ }^{4}$.

\section{Le continu constructif}

Trois illusions contribuent à l'adoption du continu classique : la " continuité " des grandeurs physiques, l'intuition géométrique, les constructions mathématiques de Cauchy, Weierstrass, Dedekind ou Cantor. Une grandeur physique n'est jamais un nombre réel, mais présente une certaine indétermination; par exemple, il n'y a pas de sens à définir la longueur d'une règle avec une erreur inférieure au rayon de l'atome. La droite réelle a des propriétés qui choquent l'intuition : il existe un ouvert de mesure $<\varepsilon$ contenant tous les rationnels contrairement aux apparences. La définition des réels par les coupures de Dedekind ou les suites de Cauchy est insuffisante, puisque, d'après le théorème de Cohen, l'hypothèse du continu ou sa négation peut être ajoutée comme axiome sans créer de contradiction. À la place du continu " classique ", nous présentons le continu constructif. La notion primitive n'est pas le réel dont la définition par les coupures de Dedekind exige une question décidable pour tout rationnel, mais le duplexe constitué par une suite de rationnels et un régulateur de convergence. Un duplexe est constitué par une suite de rationnels et un régulateur de convergence, c'est-à-dire une suite $u(n)$ de rationnels et une suitec $(n)$ d'entiers tels que :

$m, m^{\prime} \geq c(n) \Longrightarrow\left|u(m)-u\left(m^{\prime}\right)\right|<2^{-n}$

On définit la valeur absolue d'un duplexe, le maximum, le minimum, la somme, la différence, le produit de deux duplexes et, pour tout duplexe non nul, son inverse. Ces opérations ont toutes les propriétés classiques. On pose $x=0$ s'il existe une suited $(n)$ telle que :

$m \geq d(n) \Longrightarrow|u(m)|<2^{-n}$

Il faut distinguer $x \neq 0$ ( $x$ différent de 0 ), qui signifie simplement que $x$ ne peut être nul, et, $x$ \# 0 ( $x$ séparé de 0 ), qui signifie qu'il existe un entier $m$ tel que $|x|>1 / m$. La notion de duplexe équivaut à celle de suite contractante $\mathrm{d}^{\prime}$ intervalles rationnels et à celle de coupure constructive ${ }^{5}$

\section{Nombres irrationnels et transcendants}

Dès 1899, Emile Borel soulignait le caractère non constructif des démonstrations d'irrationalité et de transcendance et donnait la première mesure de transcendance de $e$. Depuis, on ne 
se contente pas d'affirmer l'irrationalité ou la transcendance de telle ou telle constante de l'analyse, mais on indique une mesure d'irrationalité ou de transcendance. Par exemple, on ne se contente pas de dire que $\pi$ ou $e^{\pi}$ est transcendant, mais on précise que, pour chaque rationnel $p / q$,

$|\pi-p / q|>q^{-42}$

$\mid e^{\pi}-p / q>q^{-c \log \log q}$

Pour presque tout réel $\alpha>1$, c'est-à-dire sauf sur un ensemble de mesure nulle, les $\alpha^{n}$ sont " bien répartis " sur le groupe additif de $R / Z$; néanmoins, un problème important et non résolu est de nommer un $\alpha$ tel que les $\alpha^{n}$ soient bien répartis. Les traités de théorie des nombres posent, et éventuellement résolvent, de nombreux problèmes d'effectivité qui, dans une optique non constructive, ne pourraient pas être posés.

Nous espérons avoir montré que l'école constructiviste, loin de renier aucun des résultats des mathématiques classiques, pose les problèmes de façon plus fine ; c'est à ce titre qu'elle demande qu'on reconnaisse l'intérêt de ses méthodes et l'importance de ses résultats ${ }^{7}$.

\section{BIBLIOGRAPHIE}

J.-P. Azra et B. Jaulin, Récursivité, Paris, Gauthier-Villars, 1973.

E. Bishop, Foundations of Constructive Analysis, New York,MacGraw Hill, 1967.

G. S. Boolos et R. C. Jeffrey, Computability and Logic, Cambridge University Press, 1967 : seconde édition revue et augmentée : 1980.

N. Bourbaki, Théorie des ensembles, Paris, Hermann, 1954-1960.

M. Davis, Computability and Unsolvability, New-York, MacGraw Hill, 1958.

R. L. Goodstein, Recursive Analysis, Amsterdam, North-Holland, 1961.

H. Hermes, Enumerability, Decidability, Computability, Heidelberg, Springer, 1965.

A. Heyting, Les Fondements des mathématiques. Intuitionnisme. Théorie de la connaissance, Paris, Gauthiers-Villars, 1955.

D. Hilbert, " Auf das Unendliche ", Mathematische Annalen, vol. 95, 1926, p. 161-190.

N. D. Jones, Computability Theory, New-York, Academic Press, 1973.

S. C. Kleene, Introduction to Metamathematics, Amsterdam, North-Holland, 1952.

S. C. Kleene et R. E. Vesley, The Foundations of Intuitionnistic Mathematics, Amsterdam, NorthHolland, 1965.

J. Loeckx, Computability and Decidability, Heidelberg, Springer, 1976.

P. Lorenzen, Einfuhrung in die operative Logik und Mathematik, Heidelberg, Springer, 1955, 1959. A. I. Malcev, Algorithms and Recursive Functions, Groningue, Wolters-Noordhoff, 1970.

A. A. Markov, Theory of Algorithms, Jerusalem, Israël Program for Scientific Translations, 1961.

V. A. Oupensky, Leçons sur les fonctions calculables, Paris, Hermann, 1966.

R. Peter, Recursive Functions, New York, Academic Press, 1967.

H. Poincaré, La Science et l'Hypothèse, Paris, Flammarion, 1902.

- La Valeur de la Science, Paris, Flammarion, 1905.

- Science et Méthode, Paris, Flammarion, 1908.

H. Rogers, Theory of Recursive Functions and Effective Computability, New York, MacGraw Hill, 1967. 
N. A. Shanin, " Constructive real numbers and constructive function spaces. Translations of mathematical monographs ", American Mathematical Society, vol. 21, 1968.

H. Weyl, Das Kontinuum, Leipzig, 1918. Réimpr. New York, Chelsea, s. d.

A. Yasuhura, Recursive Functions and Logic, New York, Academic Press, 1971.

\begin{abstract}
Notes:
1- G. Cantor, " Beiträge zur Begründung der transfinite Mengenzahlen ", Mathematische Annalen, vol. 46, 1895, p. 481. Réimprimé dans G. Cantor, Gesammelte Abhandlungen, Heidelberg, Springer-Verlag, 1932, et Hildescheim, G. Olms, 1966, p. 282.

2- Provinciales de Pascal montrent comment le double langage permet à deux groupes qui défendent des thèses opposées de s'unir pour en écraser un troisième. 3- N. Bourbaki, Théorie des ensembles, Paris, Hermann, 1970, p. E IV. 71, note 1. $4-$ Voir l'appendice 2 : " Récursivité ", p. 126 sq. (N.d.É.).

5- Une suite contractante d'intervalles est définie par une suite $d^{\prime}$ intervalles ]$u_{n}, v_{n}[$ tels que:

$\forall n, u_{n}^{<} u_{n+1}<v_{n}<v_{n+1}$ et $\forall n, \exists m,\left|v_{m}-u_{m}\right|<2^{-n}$.

6- Peut-être est-ce là le sens des travaux de certains mathématiciens qui, tels A. D. Gelfond, C. L. Siegel et A. Baker, sans professer ouvertement une philosophie constructiviste ou intuitionniste, n'en ont pas moins apporté des résultats relevant de méthodes constructives, et dont l'importance a été unanimement reconnue. Estil besoin de rappeler que, depuis la première version de ce texte, R. Apéry, à un âge où l'on n'est plus éligible pour la médaille Fields (limite d'âge : quarante ans ; pas de limite pour les prix Nobel !), a démontré l'irrationalité de $\zeta(3)$, nombre qui résistait depuis Euler à tous les efforts pour en déterminer la nature. Ce résultat étonna la communauté mathématique, au point qu'au début certains n'osèrent y croire. (N.d.É.).
\end{abstract}

En las referencias bibliográficas se puede apreciar el vasto interés matemático de Apéry, nos concentraremos sólo $\zeta(3)=\sum_{n=1}^{\infty} \frac{1}{n^{3}}$ y las anécdotas que circulan de la forma en que Apéry demostró la iracionalidad de esté número real.

Un mini recuento de varios siglos:

- 1736: Euler demuestra que $\zeta(2)=1 / 12+1 / 22+1 / 32+\ldots=\frac{\pi^{2}}{6}$

- 1737: Euler usa el símbolo $\pi$

- 1740: Euler presenta formas cerradas para $\zeta(2 n), n$ entero positivo. $\zeta(2 n)=\frac{(-1)^{n+1}}{2 *(2 n) !}(2 \pi)^{2 n} B(2 n)$, en donde $B(2 n)$ denota el número de Bernouille $2 n$

- 1761: Lambert demuestra que $\pi$ es irracional

- 1794: Legendre demuestra que $\pi^{2}$ es irracional 
- 1882: Lindemann demuestra que $\pi$ es trascendente

Alfred van der Poorten hace un recorrido sobre la demostración de Apéry de que $\zeta(3)$ es un número irracional a lo largo de varias décadas hasta afirmar ; ¡es correcta! ([6], [18]).

En [1] se cuentan algunas anéctodas de lo acontecido durante la presentación de Apéry de su demostración. En su presentación en junio 1978 en las Jornadas Aritméticas en Marseille-Lumuny hubo un cambio de programa, se indicó que Apéry(Caen) hablaría el jueves a las 2pm sobre la irracionalidad de $\zeta(3)$. Habían corrido con anterioridad rumores de este resultado, pero en general había un estado de escepticismo.

Apéry escribió $\zeta(3)=\sum_{n=1}^{\infty} \frac{1}{n^{3}}=\frac{5}{2} \sum_{n=1}^{\infty} \frac{(-1)^{n-1} n !}{n^{3}(2 n) !}$, surgieron voces de sorpresa. Alguien en la sala tenía una calculadora científica y programó las dos fórmulas hasta la capacidad del calculador y gritó: ¡es cierto!.

Se le preguntó a Apéry de donde había sacado la fórmula y este contestó: 'de mi jardín'.

La demostración es una gran proeza intelectual, Apéry presentó dos artículos sobre este tema empero para la gran mayoría de los lectores resultaron un tanto crípticos, de ahí la importancia de Van der Poorten de completar con la colaboración de muy destacados teóricos los pasos y afirmaciones en la metodología usada para demostrar la irracionalidad de $\zeta(3$.

Al día de hoy no sabemos si en general $\zeta(2 n+1)$ es un número irracional con $n$ entero positivo.

Se han obtenido resultados parciales que apuntan a que la conjetura $\zeta(2 n+1)$ )es un número irracional con $n$ entero positivo es correcta. Apéry nos indicó que a veces caminos que parecen poco ortodoxos pueden llevar a buen rumbo.

Apéry fue una persona multifacética, apasionado de las cosas que hacía, un hombre radical para bien o para mal.

\section{Bibliografía}

[1] Lipton, Regan People,Problems and Proofs. Springer-Verlag, 2013

[2] Michel Mendès-France. "Roger Apéry et l'irrationel". La Recherche, \#97, pp. 170-172, February 1979.

[3] Frits Beukers (2003). "Consequences of Apéry's work on z(3)". https://www.staff.science.uu. $\mathrm{nl} /$ beuke106/caen.pdf

[4] Pierre Ageron. "La philosophie mathématique de Roger Apéry. Philosophia Scientae, 2005 http: //philosophiascientiae.revues.org/89?lang=en

[5] François Apéry. "Roger Apéry, 1916-1994: A Radical Mathematician" The Mathematical Intelligencer, vol. 18, 2 (1996) pp 54-61.

[6] A. van der Poorten (1978). "A Proof that Euler Missed" Journées Arithmétiques de MarseilleLuminy, June. http://pracownicy.uksw.edu.pl/mwolf/Poorten_MI_195_0.pdf

[7] R. Apéry. "Mathématique constructive". Collect. Points Sér. Sci.29 (Seuil, Paris, 1982), 58-72. 
[8] R. Apéry. "Les mathématiques sont-elles une théorie pure?". Dialectica6 (1952), 309-310.

[9] R. Apéry. "Le rôle de l'intuition en mathématiques". In Congrès International de Philosophie des Sciences, Paris, 1949III (Hermann \& Cie., Paris, 1951), 85-88.

[10] R. Apéry "Axiomes et postulats". In Library of the Tenth International Congress of Philosophy, Amsterdam, 11-18 August 1948I (1949), 708-710.

[11] T. Apostol. "A proof that Euler missed: evaluating $\zeta(2)$ the easy way". The Mathematical Intelligencer 5 (3) (1983), 59-60.

[12] F. Beukers. "Consequences of Apéry's work on $\zeta(3)$ ". Preprint of talk presented at the Rencontres Arithmétiques de Caen, $\zeta(3)$ Irrationnel: Les Retombées, 1995.

[13] F. Beukers. "A note on the irrationality of $\zeta(2)$ and $\zeta(3) "$, Bull. London Math. Soc.11 (1979), 268-272.

[14] M. Black. "Review: Axiomes et postulats by Roger Apéry". The Journal of Symbolic Logic14 (3) (1949), 183.

[15] P. E. Gibbs, "'Crackpots' Who Were Right II." Prespacetime Journal1 (3) (2010), 489-497.

[16] Y. Hellegouarch. "Roger Apéry (1916-1994)". Gaz. Math. No.64 (1995), 82-83.

[17] M. Mendés-France. "Roger Apéry et l'irrationnel". La Recherche 97 (February 1979), 170-172.

[18] A. van der Poorten (1979). "A proof that Euler missed". The Mathematical Intelligencer1 (4), 195-203.

[19] N. A. Carella. "Irrationality of the Zeta Constants". https://arxiv.org/abs/1212.4082. Versión 2016

[20] J.L. Berggren, J. Borwein, P. Borwein. "Pi: A source book". Sringer-Verlag New York,2004

[21] L. Euler. Introduction to Analysis of the Infinite. Book 1. Springer-Verlag, New York, 1988-1990, pp. 137-153. (Translated from the Latin by J. D. Blanton)

[22] L. Euler. "De summis serierum reciprocarum" Comment. Acad. Sci. Petropolit., 7 (1734/35), (1740) 123-134; Opera omnia, Ser. 1 Bd. 14, 73-86. Leipzig-Berlin, 1924. 